\title{
SIMULATING THE DEMAND RESHAPING AND SUBSTITUTION EFFECTS OF PROBABILISTIC SELLING
}

\author{
Zhang, Y.*; Huang, A. Q., ${ }^{* \#}$; Cheng, T. C. E. ${ }^{* *}$; Wang, S. Y. ${ }^{* * *} \&$ Fernandez, V. ${ }^{* * *}$ \\ *Department of Logistics Management, School of Economics and Management, \\ Beijing Jiaotong University, Beijing 100044, P. R. China \\ ${ }^{* *}$ Department of Logistics and Maritime Studies, The Hong Kong Polytechnic University, \\ Hung Hom, Kowloon, Hong Kong SAR, P. R. China \\ ${ }^{* * *}$ Academy of Mathematics and Systems Science, Chinese Academy of Sciences, \\ Beijing 100044, P. R. China \\ ${ }^{* * * *}$ Department of Management, Universitat Politècnica de Catalunya. BarcelonaTech, \\ Terrassa, 08222, Spain \\ E-Mail: huanganqiang@bjtu.edu.cn ( ${ }^{\sharp}$ Corresponding author)
}

\begin{abstract}
This paper addresses the effect of probabilistic selling on inventory decisions and the expected profit through demand reshaping and demand substitution. By considering a scenario with two higher-priced specific products and one lower-priced probabilistic product, we construct a new newsboy-type inventory model with demand reshaping and substitution. A simulation study is implemented to explore extensively the effects of demand uncertainty, demand correlation, price sensitivity and price discount on the inventory decisions and profitability of probabilistic selling. Finally, we provide insightful managerial implications of the nature of inventory management mechanism of probabilistic selling.

(Received, processed and accepted by the Chinese Representative Office.)
\end{abstract}

Key Words: Inventory, Probabilistic Selling, Demand Substitution, Demand Reshaping

\section{INTRODUCTION}

It is a common business practice in travel industry to offer the customers some products with unknown information. For example, intermediaries like Priceline.com, Hotwire.com and Germanwings.com collect substitutional service products from various vendors and then pack them into probabilistic service products with some information concealed. Customers cannot know the exact information about the probabilistic products until their orders are confirmed. The success of the travel industry in deploying probabilistic selling has inspired the retailing companies like Tmall.com, jd.com, and AgonSwim.com to offer probabilistic products at lower prices with some hidden attributes, e.g., colour, pattern, style, etc. [1]. For example, a customer could buy a popular red T-shirt at a higher price or that with a random colour at a lower price.

A large body of literature has investigated the probabilistic selling from the perspective of marketing and identified the main motivation of probabilistic selling as market segmentation and price discrimination. It allows customers with weak preference for some attributes to buy what they demand at a lower price. Moreover, it enables the retailer to charge higher price from the customers with strong preference for some specific products. In addition to the above mentioned, probabilistic selling also affects the inventory management strategy. On one hand, the lower-priced probabilistic products would cannibalize the demand for the regular-priced products, which can reshape the demand and reduce aggregate demand uncertainty. On the other hand, it benefits the seller through demand substitution. For example, we assume that two specific substitute products ( $i$ and $j$, also called source products) and a probabilistic product $(k)$ composed of them $(e . g$., product $k$ is product $i$ with the 
probability of $30 \%$ and product $j 70 \%$ ) constitute the total inventory. When the demand for product $i$, denoted by $D_{i p}$, is so large that stock-out occurs, the retailer can allocate more product $j$ to satisfy the demand for product $k$, thus more product $i$ from $k$ can be used to meet $D_{i p}$. In this way, the demand for product $i$ can be substituted by product $j$ through $k$.

In traditional selling with demand substitution, the retailer is assumed to substitute one product for another in the stock-out situation [2-4]. From a new perspective, this paper investigates a scenario where the homogeneous products with different attributes like colours, shapes and materials, etc., do not directly substitute each other but through the probabilistic products. The demand for probabilistic products may come from two parts: one is the increased demand (denoted by IPD) due to lower-priced probabilistic products, and the other is the switched demand (denoted by SPD) from the demand for the regular-priced products due to price discount [5-6]. To concentrate on the effect of probabilistic selling on inventory management, we ignore the increased demand IPD.

The main purpose of this paper is to analyse the effect of probabilistic selling on the inventory management through demand substitution and demand uncertainty. We establish a newsvendor style model to characterize the demand substitution pattern and obtain some insights into inventory management. The results show that, even without considering IPD, probabilistic selling is able to benefit the seller with higher expected profit and lower inventory by reducing the demand uncertainty and increasing inventory efficiency.

The structure of the remainder is as follows: Section 2 briefly reviews the relevant literature. Section 3 presents the assumptions and definitions, based on which the inventory model with demand substitution in probabilistic selling is formulated and analysed. By using a simulation approach, Section 4 analyses the effect of probabilistic selling on inventory management and provides some insightful managerial implications. Section 5 concludes this paper.

\section{LITERATURE REVIEW}

This paper aims to study the effect of probabilistic selling with demand substitution and reshape on inventory management, and thus is closely related to three academic streams including probabilistic selling, inventory substitution, and demand reshaping.

\subsection{Probabilistic selling}

Probabilistic selling first defined by Fay and Xie means that the retailer creates an additional probabilistic product with hidden information using existing products [7]. Many studies also regard it as the similar strategy with opaque selling [8]. The studies about probabilistic selling are nascent but growing. The majority of the research focus on the marketing perspective, addressing the problems of pricing, channel selection [8], design of transparency level [9], and product mix design [10], and have demonstrated significantly positive effects of probabilistic selling on market expansion, market segmentation, price discrimination and product line extension.

It is crucial to improve inventory efficiency when the companies are increasing product categories to satisfy various customers' demands in the competitive market [11]. Probabilistic selling may become a promising strategy to decrease demand uncertainty while extending the products line according to some recent literature. However, from the operational perspective, studies are rarely found exploring the effect of probabilistic selling on managing inventory through demand substitution and demand reshaping. Although Fay and Xie have demonstrated the advantage of probabilistic selling to improve inventory utilization, they consider the uncertainty of the probability that one product is more popular than another, rather than the demand uncertainty [7, 12]. Rice also pointed out that very few studies showed 
the effectiveness of probabilistic selling when the seller is uncertain about the total category demand rather than the relative popularity of one specific item [13]. $\mathrm{Wu}$ and $\mathrm{Wu}$ considered stochastic demand in their single-product inventory model and explored the effect of opaque selling considering demand postponement. The authors showed that postponement of delivery allows the firm to use less safety stock to combat demand uncertainty [14]. However, their model assumes a deterministic demand for probabilistic products.

This paper extends the above research by modelling the demand as a normally distributed variable, which is widely used in OM research. Besides, different from [14], the demand for probabilistic products is set to be stochastic rather than deterministic. Moreover, this paper explores the effect of probabilistic selling on combating demand uncertainty and increase inventory efficiency through demand reshaping and substitution.

\subsection{Inventory substitution}

Inventory management with demand substitution has been widely investigated considering different substitution scenarios. Dutta and Chakraborty considered two products with one-way substitution which means one product can substitute for another but not the opposite [15]. [2] explored the two-way substitution scenario in which the two products can substitute for each other. Ernst and Kouvelis extended the two-product substitution scenario into three products with partial substitution [16]. The authors considered the business practice that the retailer sells products not only as independent items but also as part of the packaged product. Substitution does not exist among the independent items, but between the independent items and the packaged ones.

Similar to [16], this paper considers a scenario with three products including two specific products and a probabilistic product composed of them, where the two specific products cannot substitute for each other, but each of them can serve as a substitute for the probabilistic product.

\subsection{Demand reshaping}

When the retailer offers probabilistic goods, the retailer usually charges a lower price for probabilistic products than their source products. According to Meredith and Maki, a lowpriced brand would cannibalize sales of higher-priced brand (upward cannibalization) with enough price gap between the two brands [5]. Therefore, the price gap between the specific product and probabilistic product would reshape the demand: demand for specific products switching to probabilistic product.

Eynan and Fouque proposed the new approach called "demand reshaping", in which a company induces a portion of customers to switch to buy another product [6]. This approach is proved to be promising in improving profit with even small proportion of switching customers. Eynan and Fouque compared the efficiency of two risk pooling approaches including component commonality and demand reshaping [17]. Hsieh extended the twoproduct case in [17] and showed how a firm can take advantage of risk pooling in the threeproduct case [18].

It is notable that current studies rarely consider switch cost. However, the demand switch in probabilistic selling is price driven. Therefore, this paper employs a switch pattern similar to [18] but considers switch cost. 


\section{AN INVENTORY MODEL UNDER PROBABILISTIC SELLING}

\subsection{Problem description}

We consider a scenario where an online retailer of two homogeneous products, $i$ and $j$, creates the virtual probabilistic product $k$ and sells $i, j$ and $k$ simultaneously. In the probabilistic selling, the specific products $i$ and $j$ achieve substitution through the probabilistic product $k$ instead of being direct substitutes for each other.

The event sequence of probabilistic selling is as follows: First, the retailer purchases a quantity $Q_{i}^{p}$ of product $i$ and a quantity $Q_{j}^{p}$ of product $j$. Second, he announces the prices of specific product and the price discount of the probabilistic products. All the demand for the products arrives at the same time. Then the retailer would substitute the available products for the stock-out products. The substitution policy is that the demand for specific product $i$ and $j$ is prioritized to be satisfied. The retailer aims to make the optimal inventory decisions with different price discounts.

\subsection{Notation and assumption}

The following notations are used throughout the paper:

$p$ - the selling price of two homogeneous specific products,

$v$ - discount of the regular price $p$ (i.e., the probabilistic product $k$ would be sold at price $(1-v) p)$,

$\lambda$ - price sensitivity level of customers (the customers with higher price sensitivity are more likely to switch from purchasing the specific products to probabilistic products),

$r \quad$ - the rate of demand switching from specific product $i(j)$ to probabilistic product $k$,

$c$ - purchasing cost of specific products and probabilistic products,

$s$ - salvage cost of specific product and probabilistic product,

$q$ - penalty cost of specific product and probabilistic product,

$Q_{i}^{p}$ - purchase quantity of product $i$ in probabilistic selling,

$Q_{j}^{p}-$ purchase quantity of product $j$ in probabilistic selling,

$D_{i}$ - original stochastic demand for product $i$ without offering the probabilistic product,

$D_{j}$ - original stochastic demand for product $j$ without offering the probabilistic product,

$D_{i}^{p}$ - stochastic demand for product $i$ when offering the probabilistic product,

$D_{j}^{p}$ - stochastic demand for product $j$ when offering the probabilistic product,

$u_{i}-$ mean of the original demand $i$,

$u_{j}-$ mean of the original demand $j$,

$u_{k}-$ mean of the demand for probabilistic product $k$,

$\sigma_{i}-$ standard deviation of the original demand $i$,

$\sigma_{j}-$ standard deviation of the original demand $j$,

$\sigma_{k}$ - standard deviation of the demand for probabilistic product $k$,

$\rho_{i j}$ - the correlation coefficient of original specific demand for product $i$ and $j$, $f(x, y)$ - the joint distribution function of the original demand for specific product $i$ and $j$.

Without loss of generality, this paper assumes the demand of specific products to follow a normal distribution.

The retailer gives a discount of $v$ to induce some customers to buy the probabilistic product. When introducing the discounted probabilistic product, $r$ percent of the customers who originally intended to buy specific product $i$ would switch to buy the probabilistic product $k$. The switch rate $r$ is related to two key elements including the price gap and the level of product differentiation [5]. Therefore, the switch rate can be defined as: 


$$
r=\lambda v
$$

where $\lambda$ is defined as the price sensitivity level of customers. With a certain price discount $v$, the larger value of $\lambda$ also indicates weak product differentiation. The discount rate $v$ satisfies $0 \leq v<1-c / p$ to make sure that the price of the probabilistic product is higher than the cost $c$. Eq. (1) implies that the switch rate is positively related to the discount $v$ when $\lambda>0$. When $r=0$, the probabilistic selling problem degenerates into a classic newsvendor problem without demand substitution and into centralized inventory strategy when $r=1$.

\subsection{Demand reshaping function}

We assume that the total demand for probabilistic products come from demand for the specific source products. Thus, the original demand for the two specific products $i$ and $j$ would be reshaped in probabilistic selling as follows:

$$
\begin{aligned}
& D_{i}^{p}=(1-r) D_{i}, \\
& D_{j}^{p}=(1-r) D_{j} .
\end{aligned}
$$

The demand distribution of the probabilistic product can be expressed as:

$$
D_{k}=r D_{i}+r D_{j} .
$$

\subsection{Expected profit function}

The expected profit in Eq.(4) includes the revenue, the salvage cost, the penalty cost and the acquisition cost.

$$
\begin{aligned}
& E\left(Q_{i}^{p}, Q_{j}^{p}\right)=E\left\{p \min \left(D_{i}^{p}, Q_{i}^{p}\right)+p \min \left(D_{j}^{p}, Q_{j}^{p}\right)\right. \\
& +(1-\lambda v) p \min \left[D_{k},\left[\left(Q_{i}^{p}-D_{i}^{p}\right)^{+}+\left(Q_{j}^{p}-D_{j}^{p}\right)^{+}\right]\right] \\
& +s\left[\left(Q_{i}^{p}-D_{i}^{p}\right)^{+}+\left(Q_{j}^{p}-D_{j}^{p}\right)^{+}-D_{k}\right]-\mathrm{c}\left(Q_{i}^{p}+Q_{j}^{p}\right) \\
& \left.\left.-q\left[\left(D_{i}^{p}-Q_{i}^{p}\right)^{+}+\left(D_{j}^{p}-Q_{j}^{p}\right)^{+}+\left(D_{k}-\left(Q_{i}^{p}-D_{i}^{p}\right)^{+}-\left(Q_{j}^{p}-D_{j}^{p}\right)^{+}\right)^{+}\right]\right]\right\}
\end{aligned}
$$

The demand for the products varies over the domain $D_{i} \geq 0$ and $D_{j} \geq 0$. With a given switch rate $r \geq 0$, the expected profit $E\left(Q_{i}^{p}, Q_{j}^{p}\right)$ depends on the relationship between $r$ and the optimal order size $Q_{i}^{p}$ and $Q_{j}^{p}$. Therefore, the expected profit can be calculated in four different cases, as shown in Fig. 1.

Considering the spirits of modelling the expected profit are the same in the above four cases, this section just presents the details of case IV for conciseness. The expected profit functions in the other three cases can be formulated in the same way. Analysis of Case IV proceeds as follows:

(1) In the condition of $\left\{Q_{i} \geq(1-r) \mathrm{D}_{i}, Q_{j} \geq(1-r) \mathrm{D}_{j}, Q_{i}+Q_{j}-\mathrm{D}_{i}-\mathrm{D}_{j} \geq 0\right\}$, the probabilistic product is out of stock. However, the generated substitute demand for product $i$ (or product $j$ ) can be met from the shelf. The expected profit function is:

$$
\int_{0}^{Q_{i}^{p}+Q_{j}^{p}} \int_{0}^{Q_{i}^{p}+Q_{j}^{p}-x}\left[\begin{array}{l}
p(1-r) x+p(1-r) y+(1-v) p(r x+r y)+s\left(Q_{i}^{p}+Q_{j}^{p}-x-y\right) \\
-c\left(Q_{i}^{p}+Q_{j}^{p}\right)
\end{array}\right] f(x, y) d x d y .
$$



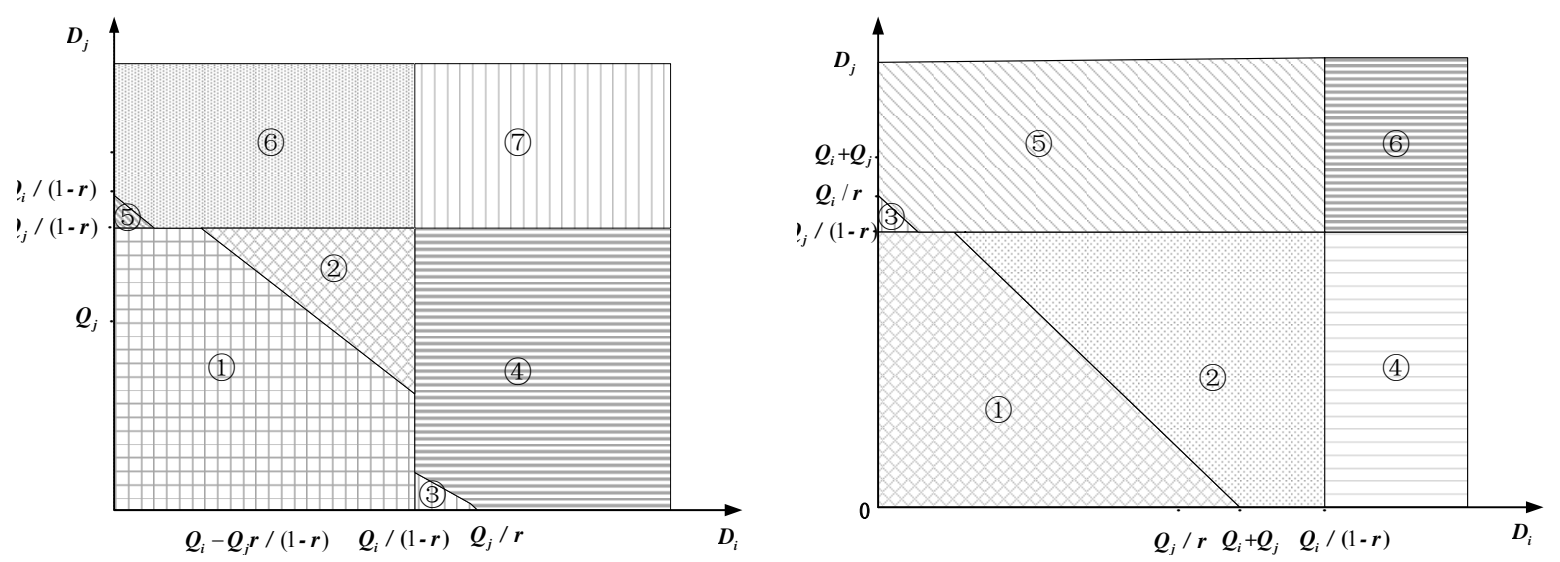

Case I: $r Q_{i}^{p}+r Q_{j}^{p} \leq Q_{i}^{p}$ and $r Q_{i}^{p}+r Q_{j}^{p} \leq Q_{j}^{p}$

Case II: $r Q_{i}^{p}+r Q_{j}^{p} \leq Q_{i}^{p}$ and $r Q_{i}^{p}+r Q_{j}^{p} \geq Q_{j}^{p}$
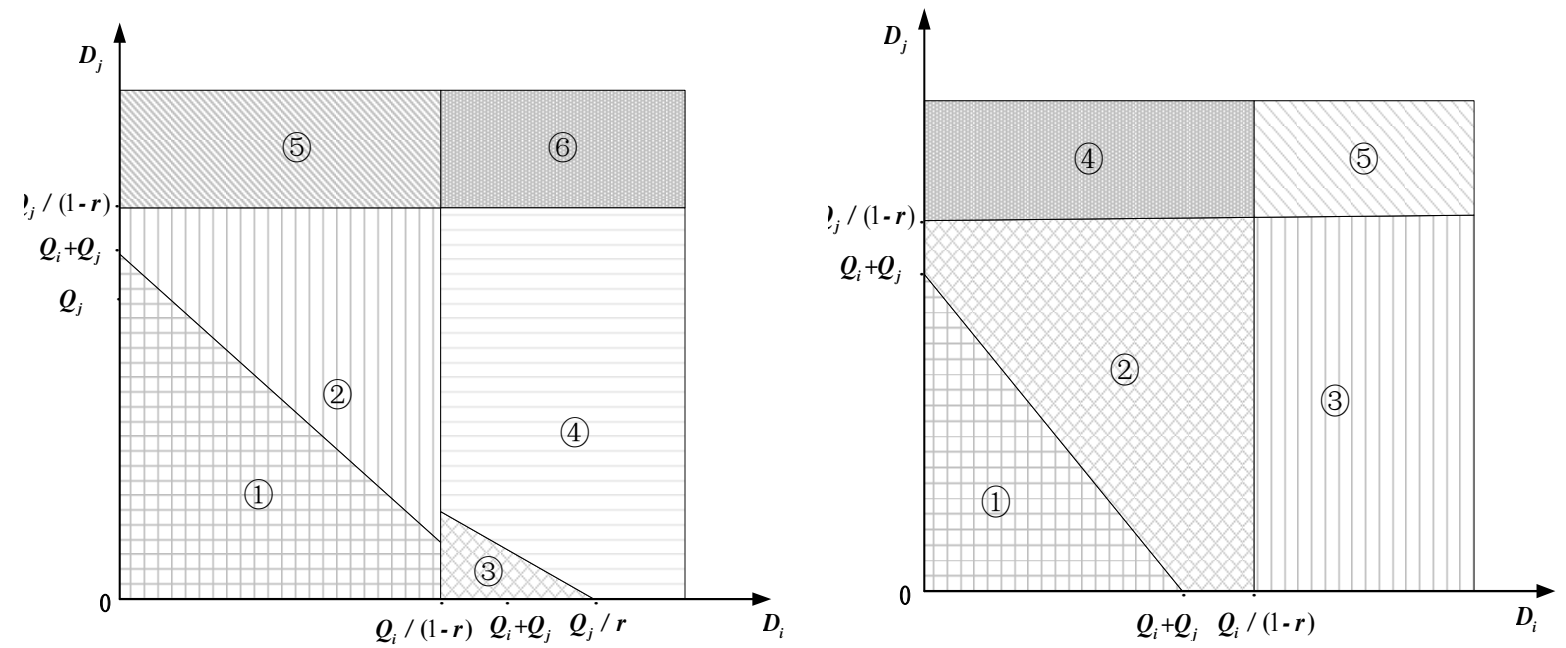

Case III: $r Q_{i}^{p}+r Q_{j}^{p} \geq Q_{i}^{p}$ and $r Q_{i}^{p}+r Q_{j}^{p} \leq Q_{j}^{p} \quad$ Case IV: $r Q_{i}^{p}+r Q_{j}^{p} \geq Q_{i}^{p}$ and $r Q_{i}^{p}+r Q_{j}^{p} \geq Q_{j}^{p}$

Figure 1: The four cases of the expected profit.

(2) In the condition of $Q_{i} \geq(1-r) \mathrm{D}_{i}, Q_{j} \geq(1-r) \mathrm{D}_{j}, Q_{i}+Q_{j}-\mathrm{D}_{i}-\mathrm{D}_{j} \leq 0$, the probabilistic product is stock out. Only one part of the substitute demand can be met from the remaining inventory of products $i$ and $j$. The relevant expected profit function is:

$$
\begin{array}{r}
\int_{0}^{Q_{i}^{p}+Q_{j}^{p}} \int_{Q_{i}^{p}+Q_{j}^{p}-x}^{\frac{Q_{j}^{p}}{1-r}}\left[\begin{array}{l}
p(1-r) x+p(1-r) y+(1-v) p\left(Q_{i}^{p}+Q_{j}^{p}-(1-r) x-(1-r) y\right) \\
-q\left(x+y-Q_{i}^{p}-Q_{j}^{p}\right)-c\left(Q_{i}^{p}+Q_{j}^{p}\right)
\end{array}\right] f(x, y) d x d y \\
+\int_{Q_{i}^{p}+Q_{j}^{p}}^{\frac{Q_{i}^{p}}{1-r}} \int_{0}^{\frac{Q_{j}^{p}}{1-r}}\left[\begin{array}{l}
p(1-r) x+p(1-r) y+(1-v) p\left(Q_{i}^{p}+Q_{j}^{p}-(1-r) x-(1-r) y\right) \\
-q\left(x+y-Q_{i}^{p}-Q_{j}^{p}\right)-c\left(Q_{i}^{p}+Q_{j}^{p}\right)
\end{array}\right] f(x, y) d x d y .
\end{array}
$$

(3) In the condition of $Q_{i} \leq(1-r) \mathrm{D}_{i}, Q_{j} \geq(1-r) \mathrm{D}_{j}, Q_{j}-(1-r) \mathrm{D}_{j} \leq \mathrm{D}_{i}+\mathrm{D}_{j}$, both the probabilistic product and specific product $i$ are stock out. Furthermore, only one part of the substitute demand can be met from the remaining inventory of product $j$. The relevant expected profit function is:

$\int_{\frac{Q_{i}^{p}}{1-r}}^{\infty} \int_{0}^{\frac{Q_{j}^{p}}{1-r}}\left[\begin{array}{l}p Q_{i}^{p}+p(1-r) y+(1-v) p\left[Q_{j}^{p}-(1-r) y\right]-q\left(x+y-Q_{i}^{p}-Q_{j}^{p}\right) \\ -c\left(Q_{i}^{p}+Q_{j}^{p}\right)\end{array}\right] f(x, y) d x d y$. 
(4) In the condition of $Q_{i} \leq(1-r) \mathrm{D}_{i}, Q_{j} \leq(1-r) \mathrm{D}_{j}$, both the specific products $i$ and $j$ are stock out. None of the substitute demand can be met. The relevant expected profit function is:

$\int_{\frac{Q_{i}^{p}}{1-r}}^{\infty} \int_{\frac{Q_{j}^{p}}{1-r}}^{\infty}\left[p Q_{i}^{p}+p Q_{j}^{p}-q\left(x+y-Q_{i}^{p}-Q_{j}^{p}\right)-c\left(Q_{i}^{p}+Q_{j}^{p}\right)\right] f(x, y) d x d y$.

(5) In the condition of $Q_{i} \geq(1-r) \mathrm{D}_{i}, Q_{j} \leq(1-r) \mathrm{D}_{j}, Q_{i}-(1-\mathrm{r}) \mathrm{D}_{i} \leq r \mathrm{D}_{i}+r \mathrm{D}_{j}$, both the probabilistic product and specific product $j$ are stock out. Furthermore, only one part of the substitute demand can be met from the remaining inventory of product $i$. The relevant expected profit function for this part is:

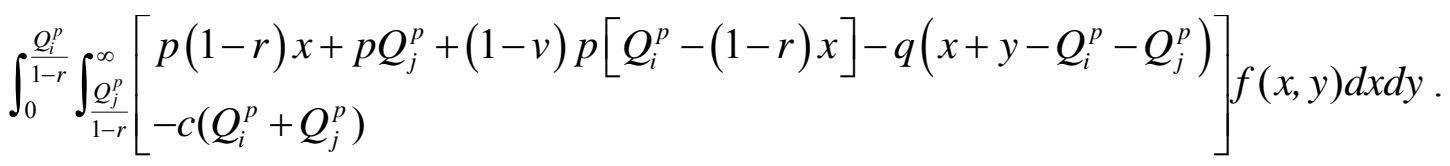

\subsection{Solution of the inventory model}

After calculating the Hessian Matrix under the four cases, we have proved that the expected function $E\left(Q_{i}^{p}, Q_{j}^{p}\right)$ is concave with reference to $\left(Q_{i}^{p}, Q_{j}^{p}\right)$. However, we cannot formulate out the closed-form solution owing to the high complexity of the expected profit function, which blocks us from deeply investigating the relationship among different variables. Fortunately, the expected profit function is concave, which enables us to search the optimal solution using a numerical simulation approach.

\section{SIMULATION INSIGHTS ON INVENTORY AND PROFIT PERFORMANCE IN PROBABILISTIC SELLING}

\subsection{Simulation design}

This simulation aims to obtain insights into the inventory and profit performance in probabilistic selling by addressing the following two questions: (1) What is the performance of probabilistic selling on total inventory level and expected profit compared with the classic newsvendor model? (2) What is the effect of demand uncertainty, demand correlation, price discount and price sensitivity on the profitability of probabilistic selling?

Consistent with the aforementioned assumptions, the parameter values are set as follows: $\mu_{i}=\mu_{j}=1200, p=40, c=10, s=0$ and $q=4, \sigma=\sigma_{1}=\sigma_{2}=\{400,500,600\}, \rho_{i j}=\{-0.5,0,0.5\}$, $v \in[0,0,75]$ and $\lambda \in[0,4 / 3]$. With different parameter combinations, we use the Mathematica to solve the optimal order of produce $i$ and $j\left(Q_{i}^{p}\right.$ and $\left.Q_{j}^{p}\right)$ and the corresponding expected profit $E\left(Q_{i}^{p}, Q_{j}^{p}\right)$. Then the profit increase $(P I)$ and inventory decrease $(D I)$ can be calculated by $P I=\frac{\left(Q_{i}^{c}+Q_{j}^{c}\right)-\left(Q_{i}^{p}+Q_{j}^{p}\right)}{Q_{i}^{c}+Q_{j}^{c}}$ and $D I=\frac{E\left(Q_{i}^{p}+Q_{j}^{p}\right)-E\left(Q_{i}^{c}+Q_{j}^{c}\right)}{E\left(Q_{i}^{c}+Q_{j}^{c}\right)}$, where $Q_{i}^{c}\left(Q_{j}^{c}\right)$ denotes the optimal order of product $i(j)$ in the classical newsvendor model.

\subsection{Simulation analysis}

\section{(1) Effects of price discount and demand uncertainty}

As shown in Fig. 2, the inventory level decreases with the price discount. This pattern can be explained from two aspects. On one hand, the increasing price discount induces more customers who intended to buy the two different specific products to buy the single probabilistic product, which reshapes the demand distribution. Consequently, the aggregate 
demand uncertainty decreases with the switching amount [6]. Besides, the demand substitution also contributes to inventory decrease. Because with a non-zero price discount, the demand for the probabilistic product becomes a pool for substitution when stock-out occurs, which also helps reduce demand uncertainty. However, with increasing price discount, the retailer would sell more lower-priced probabilistic products to customers. This is a trade-off between the benefits of the decreased inventory and profit decrease resulting from a number of low-priced customers. Therefore, the curves in Fig. 3 show that the expected profit increases with price discount first and then decreases with it. With lower price discount between 0 and 0.2 , probabilistic selling can benefit the retailer with higher profit and lower inventory. However, with a larger price discount, the benefit of lower inventory would not offset the profit decrease due to lower price, resulting in rapid decline. Another observation from Figs. 2 and 3 is that the efficiency of probabilistic selling would increase with respect to higher demand uncertainty. As demand reshaping and demand substitution would benefit the retailer with reduced demand uncertainty, there will be more room for improvement through probabilistic selling.

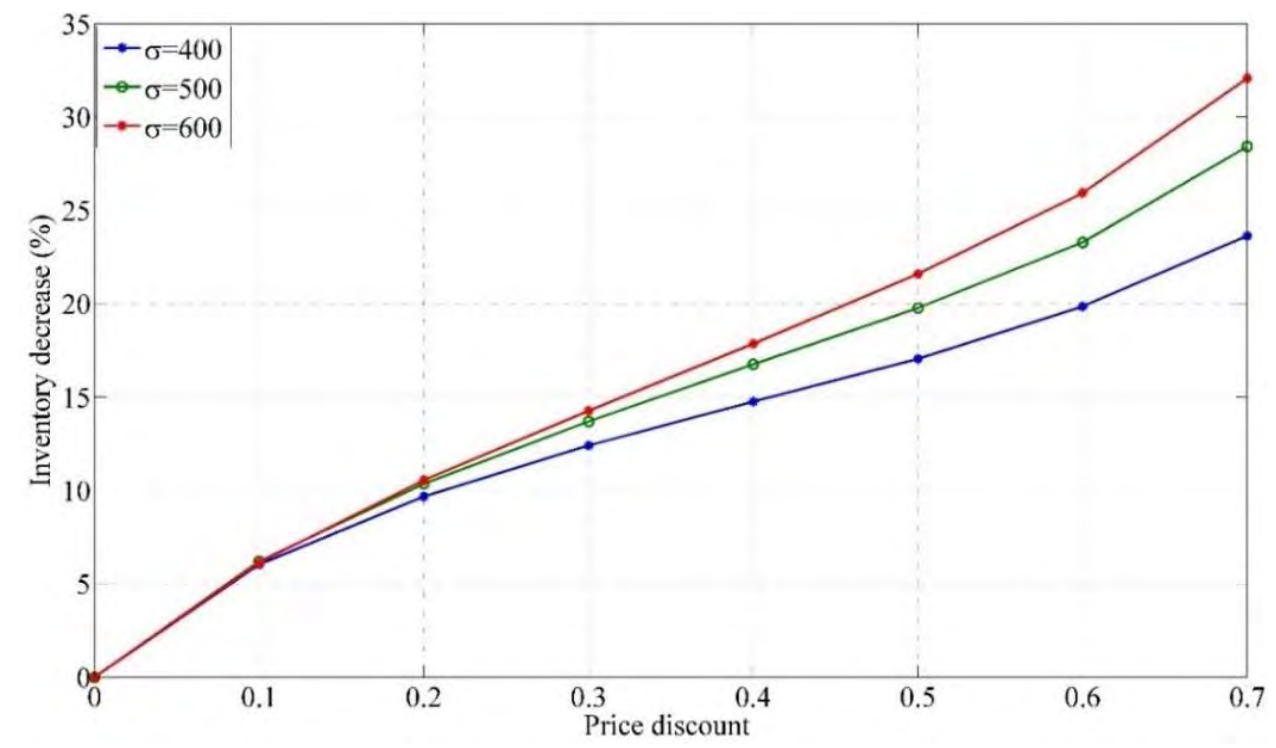

Figure 2: The effects of price discount and demand uncertainty on inventory level.

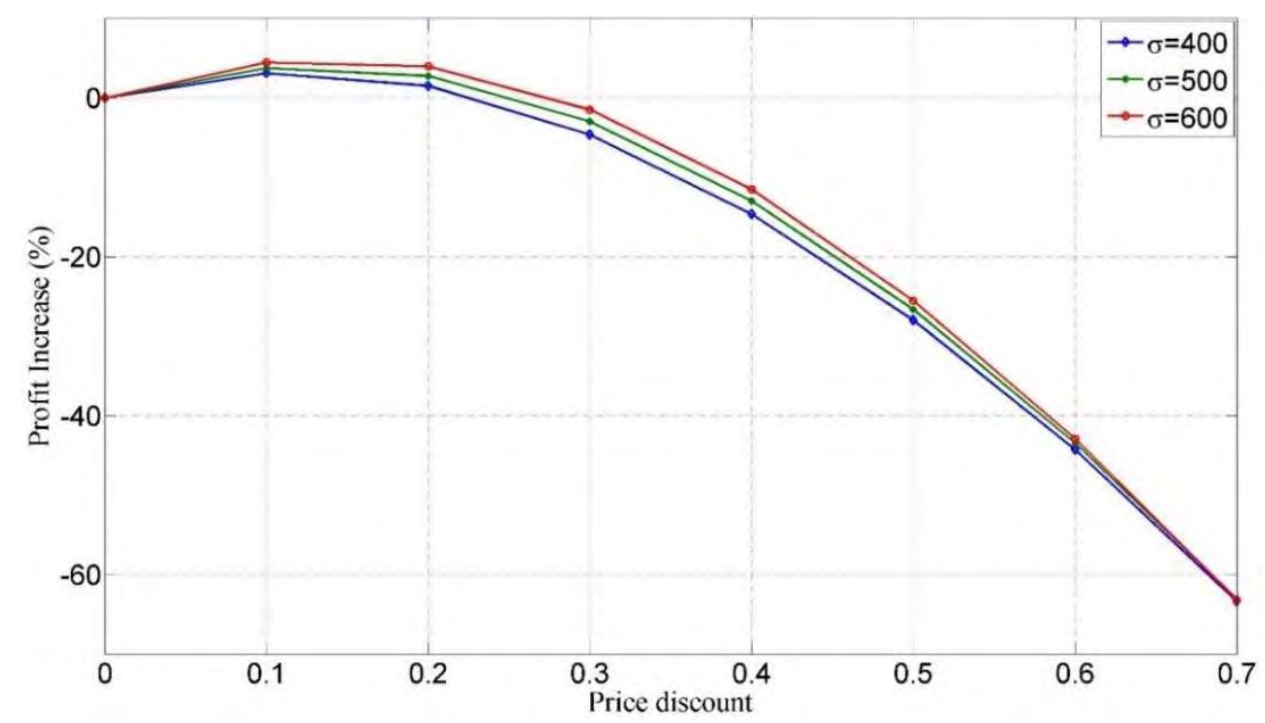

Figure 3: The effects of price discount and demand uncertainty on the expected profit. 


\section{(2) Effects of customer price sensitivity and price discount}

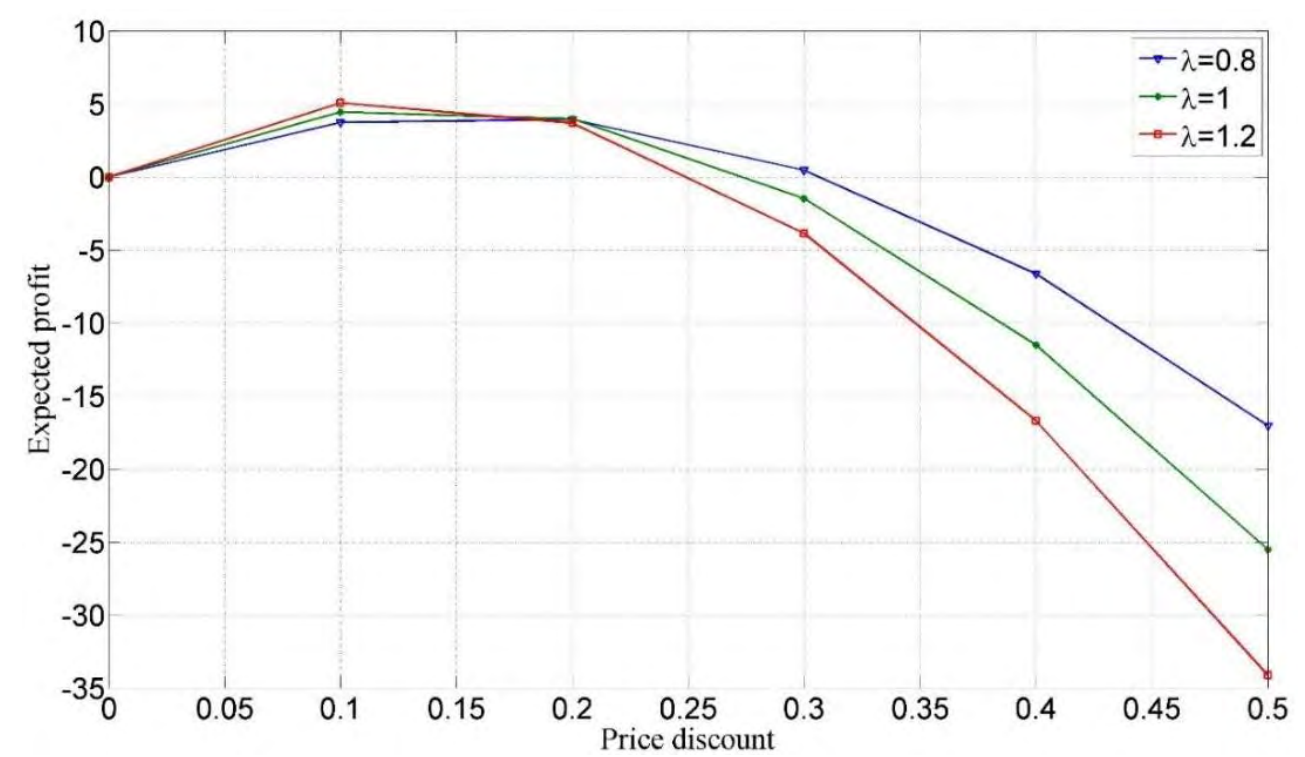

Figure 4: The effects of price sensitivity on the expected profit.

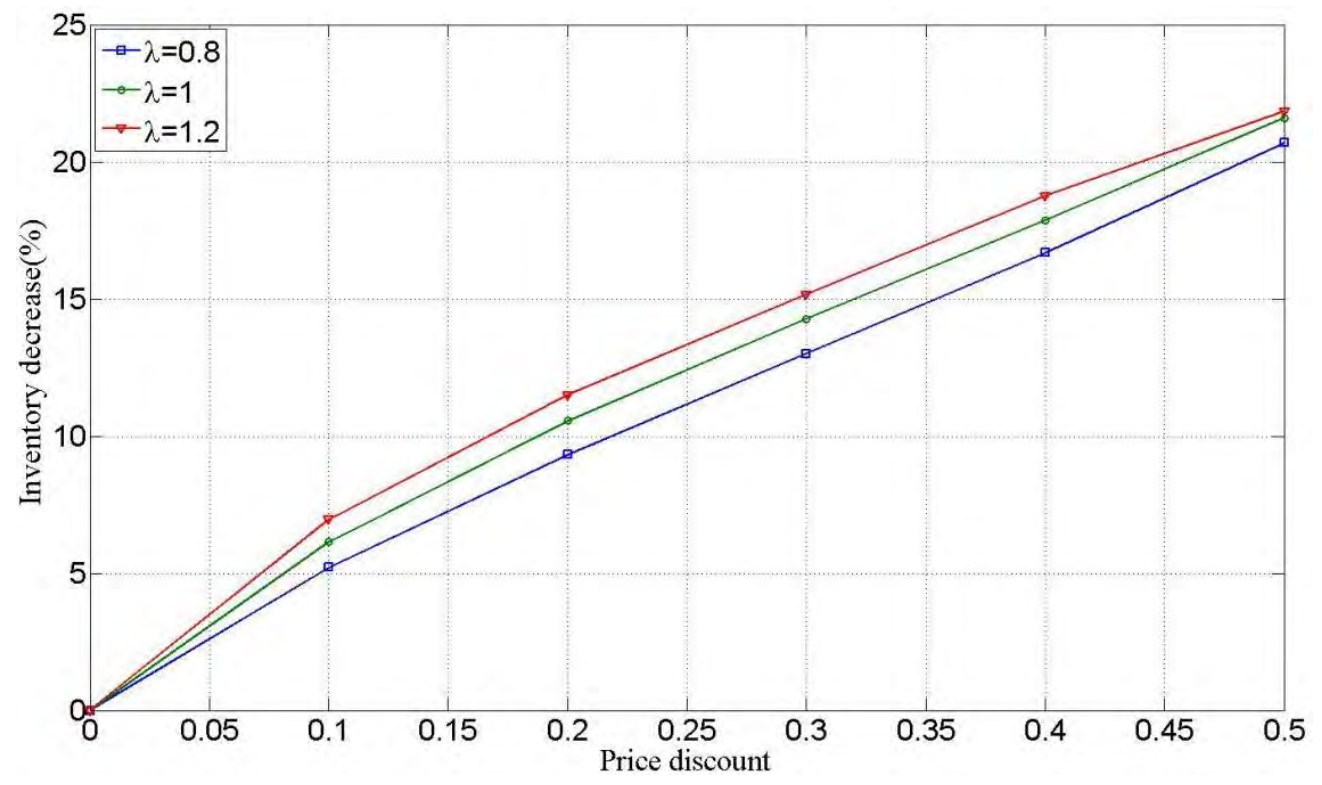

Figure 5: The effects of price sensitivity on the inventory level.

As shown in Fig. 4, when the customers' price sensitivity is higher, the retailer can achieve higher profit increase with the optimal price discount. This is logical, because with higher customers' price sensitivity, there would be more switch demand, even though the retailer offers a small price discount. In other words, the retailer will benefit more from smaller "price sacrifice". The curves in Fig. 5 share the same logic that the retailer can always get higher inventory decrease with higher customer price sensitivity. When switch rate approaches 1 , we can expect that probabilistic selling is reduced to centralized inventory and the inventory levels would be the same. We can also interpret the observation from the perspective of product differentiation. It is reasonable that lower product differentiation leads to higher price sensitivity with certain price discounts. According to the observation that higher price sensitivity can benefit the retailer with higher profits, we can conclude that the retailer has the potential to gain more profit by offering products with weaker product differentiation at the optimal price discount. 


\section{(3) Effects of demand correlation and price discount}

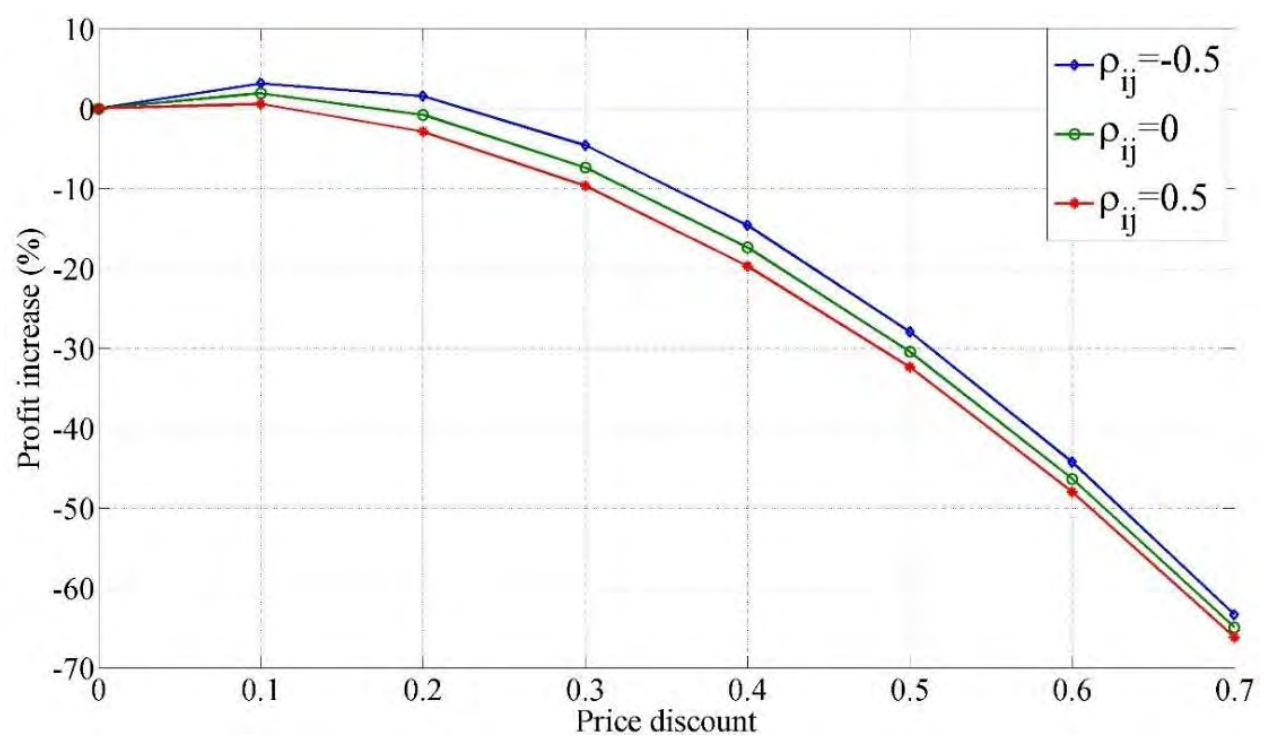

Figure 6: The effects of demand correlation on the expected profit.

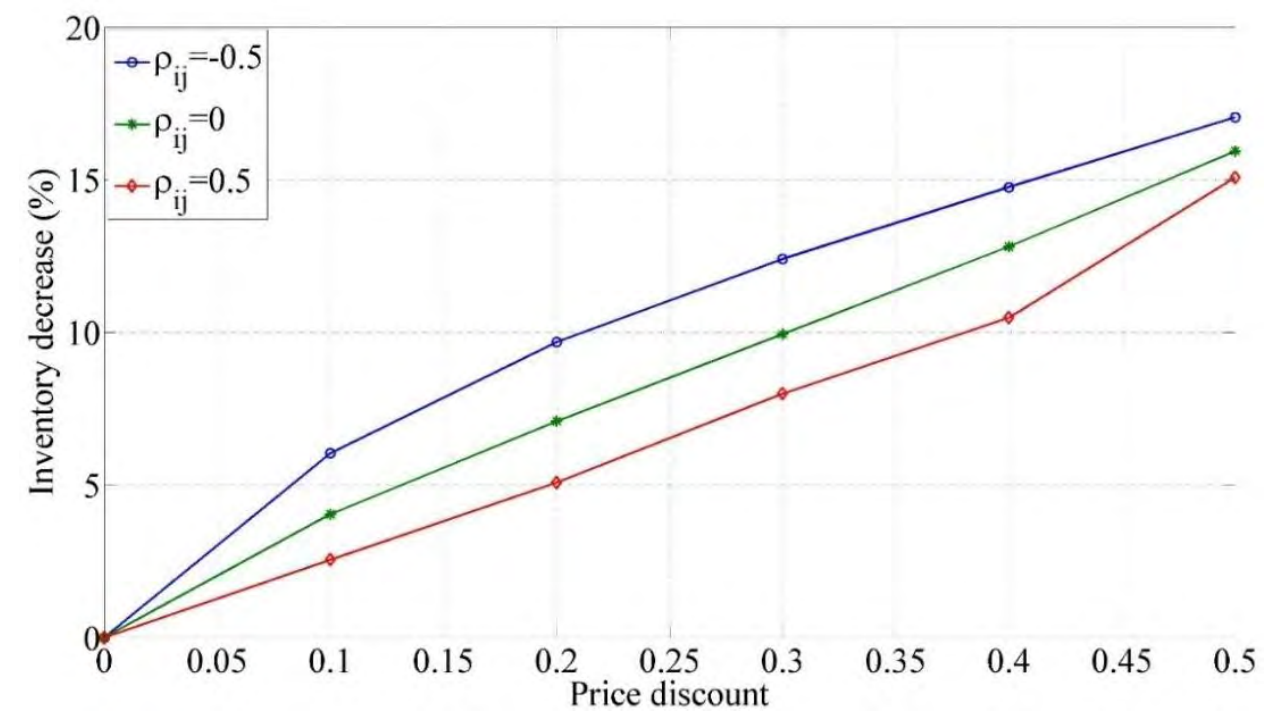

Figure 7: The effects of demand correlation on the inventory level.

From Fig. 6 we can see that the expected profit decreases with demand correlation, which is consistent with [4]. After demand reshaping, the demand for the probabilistic products is correlated with that of the specific products. The positively correlated demands of products $i$ and $j$ result in simultaneous large or small substitution demands with a high possibility. The probability that the retailer substitutes specific products for the probabilistic product occurs is low. We find in Fig. 7 that the inventory level would decrease with demand correlation. When the price discount is large enough to induce adequate demand for probabilistic products, the generated substitute demand from the regular-priced products can be satisfied. After that, when the price discount becomes larger, the benefit from demand substitution would diminish and the demand reshaping effect is more powerful.

\section{CONCLUSIONS}

In order to investigate the effect of probabilistic selling on inventory decisions and expected profit through demand reshaping and demand substitution, this paper first analyses the 
demand reshaping and substitution under probabilistic selling and proposes a single-period newsvendor model with stochastic demand for multiple products. Subsequently, a simulation experiment is implemented to figure out the effect of price discount, customer price sensitivity, the demand uncertainty and demand correlation on the retailer's optimal inventory level and expected profit. The results show that probabilistic selling can benefit the seller with higher expected profit and lower inventory by reducing the demand uncertainty and improving inventory efficiency, even without considering the increased demand due to offering the low-priced probabilistic products. Besides, the efficiency of probabilistic selling on managing inventory is closely dependent on customers' price sensitivity or product differentiation, the demand uncertainty and the demand correlation. Moreover, the effect of probabilistic selling is more significant with lower product differentiation, higher customers' price sensitivity, and higher demand uncertainty. It is noted that the optimal selection of the price discount $v$ is necessary to secure the performance of the probabilistic selling, given that unsuitable values of $v$ lead to lower expected profits in our simulation.

\section{ACKNOWLEDGEMENTS}

This research was supported in part by the NSFC under grant numbers 71390334 and 71532002 , Beijing Philosophy and Social Science Foundation under grant number 13JGB042, and the Fundamental Research Funds for the Central Universities under grant number 2015jbwy011. Cheng was also supported in part by The Hong Kong Polytechnic University under the Fung Yiu King Wing Hang Bank Endowed Professorship in Business Administration.

\section{REFERENCES}

[1] Fay, S.; Xie, J. (2010). The economics of buyer uncertainty: Advance selling vs. probabilistic selling, Marketing Science, Vol. 29, No. 6, 1040-1057, doi:10.1287/mksc.1100.0576

[2] Parlar, M.; Goyal, S. K. (1984). Optimal ordering decisions for two substitutable products with stochastic demands, OPSEARCH, Vol. 21, No. 1, 1-15

[3] Smith, S. A.; Agrawal, N. (2000). Management of multi-item retail inventory systems with demand substitution, Operations Research, Vol. 48, No. 1, 50-64

[4] Netessine, S.; Rudi, N. (2003). Centralized and competitive inventory models with demand substitution, Operations Research, Vol. 51, No. 2, 329-335

[5] Meredith, L.; Maki, D. (2001). Product cannibalization and the role of prices, Applied Economics, Vol. 33, No. 14, 1785-1793, doi:10.1080/00036840010015769

[6] Eynan, A.; Fouque, T. (2003). Capturing the risk-pooling effect through demand reshape, Management Science, Vol. 49, No. 6, 704-717, doi:10.1287/mnsc.49.6.704.16020

[7] Fay, S.; Xie, J. (2008). Probabilistic goods: A creative way of selling products and services, Marketing Science, Vol. 27, No. 4, 674-690, doi:10.1287/mksc.1070.0318

[8] Anderson, C. K.; Xie, X. (2014). Pricing and market segmentation using opaque selling mechanisms, European Journal of Operational Research, Vol. 233, No. 1, 263-272, doi:10.1016/j.ejor.2013.08.018

[9] Post, D.; Spann, M. (2012). Improving airline revenues with variable opaque products: "Blind booking" at Germanwings, Interfaces, Vol. 42, No. 4, 329-338, doi:10.1287/inte.1110.0591

[10] Fay, S.; Xie, J.; Feng, C. (2015). The effect of probabilistic selling on the optimal product mix, Journal of Retailing, Vol. 91, No. 3, 451-467, doi:10.1016/j.jretai.2015.01.004

[11] Qi, Y.-N.; Tang, M.-C.; Zhang, M. (2014). Mass customization in flat organization: The mediating role of supply chain planning and corporation coordination, Journal of Applied Research and Technology, Vol. 12, No. 2, 171-181, doi:10.1016/S1665-6423(14)72333-8

[12] Fay, S.; Xie, J. (2014). Timing of product allocation: Using probabilistic selling to enhance inventory management, Management Science, Vol. 61, No. 2, 474-484, doi:10.1287/ $\underline{\text { mnsc. } 2014.1948}$ 
[13] Rice, D. H.; Fay, S. A.; Xie, J. (2014). Probabilistic selling vs. markdown selling: Price discrimination and management of demand uncertainty in retailing, International Journal of Research in Marketing, Vol. 31, No. 2, 147-155, doi:10.1016/j.ijresmar.2013.08.006

[14] Wu, Z.; Wu, J. (2015). Price discount and capacity planning under demand postponement with opaque selling, Decision Support Systems, Vol. 76, 24-34, doi:10.1016/j.dss.2015.02.002

[15] Dutta, P.; Chakraborty, D. (2010). Incorporating one-way substitution policy into the newsboy problem with imprecise customer demand, European Journal of Operational Research, Vol. 200, No. 1, 99-110, doi:10.1016/j.ejor.2008.12.011

[16] Ernst, R.; Kouvelis, P. (1999). The effects of selling packaged goods on inventory decisions, Management Science, Vol. 45, No. 8, 1142-1155, doi:10.1287/mnsc.45.8.1142

[17] Eynan, A.; Fouque, T. (2005). Benefiting from the risk-pooling effect: internal (component commonality) vs. external (demand reshape) efforts, International Journal of Services and Operations Management, Vol. 1, No. 1, 90-99, doi:10.1504/IJSOM.2005.006320

[18] Hsieh, Y.-J. (2011). Demand switching criteria for multiple products: An inventory cost analysis, Omega, Vol. 39, No. 2, 130-137, doi:10.1016/j.omega.2010.04.004 